\title{
The Johnson cokernel and the Enomoto-Satoh invariant
}

\author{
JAMES CONANT
}

\begin{abstract}
We study the cokernel of the Johnson homomorphism for the mapping class group of a surface with one boundary component. A graphical trace map simultaneously generalizing trace maps of Enomoto and Satoh and Conant, Kassabov and Vogtmann is given, and using technology from the author's work with Kassabov and Vogtmann, this is is shown to detect a large family of representations which vastly generalizes series due to Morita and Enomoto and Satoh. The Enomoto-Satoh trace is the rank-1 part of the new trace, and it is here that the new series of representations is found. The rank-2 part is also investigated, though a fuller investigation of the higher-rank case is deferred to another paper.
\end{abstract}

17B40; 20C15, 20F28

\section{Introduction}

The Johnson homomorphism is an injective Lie algebra homomorphism $\tau: \mathrm{J} \rightarrow \mathrm{D}(H)$ (see Johnson [10] and Morita [13]), where $J$ is the associated graded Lie algebra coming from the Johnson filtration of the mapping class $\operatorname{group} \operatorname{Mod}(g, 1)$ and $\mathrm{D}(H)=$ $\mathrm{D}\left(H_{1}\left(\Sigma_{g, 1} ; \mathbb{k}\right)\right)$ is a Lie algebra of "symplectic derivations" of the free Lie algebra $\mathrm{L}(H)$. It is an isomorphism in order $1: \mathrm{J}_{1} \cong \mathrm{D}_{1}(H) \cong \bigwedge^{3} H$, and in fact a theorem of Hain [8] says that $\tau(\mathrm{J})$ is generated as a Lie algebra by the order-1 part $\wedge^{3} H$. In general, $\tau$ is not surjective and the Johnson cokernel $\mathrm{C}_{s}=\mathrm{D}_{S}(H) / \tau\left(\mathrm{J}_{S}\right)$ is an interesting $\mathrm{Sp}(H)$-module. (See Figure 1 for the known decomposition in low degrees.)

Ultimately, one would like to determine the structure of $J$, which gives information about the mapping class group. The larger Lie algebra $\mathrm{D}(H)$ is in some sense easier to understand, and for the purposes of this investigation can be considered "known". (For example, the dimensions of $\mathrm{D}_{S}(H)$ are easily calculated.) From this perspective, identifying the unknown $J$ is the same as identifying the cokernel $C$. Indeed, in Morita's 1999 survey article [14], he listed a series of problems indicating future directions of research in the mapping class group. One of these problems was to determine exactly how $\mathrm{J}$ includes into $\mathrm{D}(H)$ as an $\mathrm{Sp}$-module, and in particular, to characterize the cokernel of the Johnson homomorphism. Besides the direct application to the structure of the Johnson filtration of the mapping class group, another source 
of interest in this problem comes from number theory. Nakamura [16] showed that certain obstructions coming from the Galois group $\operatorname{Gal}(\overline{\mathbb{Q}} / \mathbb{Q})$ appear in the cokernel in even orders $2 k$. Deligne's motivic conjecture would imply that these obstructions appear with multiplicity given by the degree- $k$ part of the free graded Lie algebra $\mathrm{L}\left(\sigma_{3}, \sigma_{5}, \sigma_{7}, \ldots\right)$ with one generator in each odd degree greater than or equal to 3 . All of the representations coming from this so-called "Galois obstruction" appear as the trivial Sp-representation $[0]_{\mathrm{Sp}}$, giving an infinite family of cokernel obstructions. In addition to these somewhat mysterious Galois obstructions, two other infinite families of obstructions that are known: Morita [13] showed that representations $[k]_{\mathrm{Sp}}$ appear in the cokernel for all odd $k \geq 3$, and more recently Enomoto and Satoh [6] showed that representations $\left[1^{4 m+1}\right]_{S p}$ appear in the cokernel as well. (See Enomoto and Enomoto [5] for even more recent progress.)

In this paper, we introduce a new invariant for detecting the cokernel,

$$
\operatorname{Tr}^{\mathrm{C}}: \mathrm{C}_{s} \rightarrow \bigoplus_{r \geq 1} \Omega_{s+2-2 r, r}(H),
$$

which simultaneously generalizes the construction of Enomoto and Satoh [6] and of Conant, Kassabov and Vogtmann [4]. (The superscript "C" stands for "cokernel".) The space $\Omega_{s+2-2 r, r}(H)$ is defined as a quotient of the dimension-1 part of the hairy graph complex [4] by certain relators, shown on the right of Figure 3. The set of relations is large enough so that $\operatorname{Tr}^{\mathrm{C}}$ vanishes on iterated brackets of order-1 elements, but not so large as to project all the way down to the first homology of the hairy graph complex. The two indices $s+2-2 r$ and $r$ refer to the number of hairs and rank of the graph, respectively.

By projecting to the summands $\Omega_{s+2-2 r, r}(H)$ for fixed rank $r$, one gets a series of invariants, which we now discuss.

\subsection{Rank 1}

In Section 4, we show that the $r=1$ part $\Omega_{s, 1}(H)$ is isomorphic to $\left[H^{\otimes s}\right]_{D_{2 s}}$ and that $\operatorname{Tr}^{\mathrm{C}}$ projects to the Enomoto-Satoh trace $\operatorname{Tr}^{\mathrm{ES}}: \mathrm{C}_{s} \rightarrow\left[H^{\otimes s}\right]_{D_{2 s}}$ (Theorem 4.2). (Although their trace takes values in $\left[H^{\otimes s}\right]_{\mathbb{Z}_{s}}$, it possesses an extra $\mathbb{Z}_{2}$ symmetry.)

Let $H^{\langle s\rangle} \subset H^{\otimes s}$ be the intersection of the kernels of all the pairwise contractions $H^{\otimes s} \rightarrow H^{\otimes(s-2)}$. Then there is a projection $\pi: \Omega_{s+2-2 r, r}(H) \rightarrow \Omega_{s+2-2 r, r}\langle H\rangle$, where the latter space is defined by "taking coefficients in $H^{\langle s+2-2 r\rangle}$ ". A theorem of [3] implies that the composition $\pi \circ \operatorname{Tr}^{\mathrm{C}}$ is onto. Considering the case $r=1$ gives us the following theorem, which is one of the main results of this paper. 


$$
\begin{aligned}
\mathrm{C}_{1} & =\mathrm{C}_{2}=0 \\
\mathrm{C}_{3} & =[3]_{\mathrm{Sp}} \\
\mathrm{C}_{4} & =\left[21^{2}\right]_{\mathrm{Sp}} \oplus[2]_{\mathrm{Sp}} \\
\mathrm{C}_{5} & =[5]_{\mathrm{Sp}} \oplus[32]_{\mathrm{Sp}} \oplus\left[2^{2} 1\right]_{\mathrm{Sp}} \oplus\left[1^{5}\right]_{\mathrm{Sp}} \oplus 2[21]_{\mathrm{Sp}} \oplus 2\left[1^{3}\right]_{\mathrm{Sp}} \oplus 2[1]_{\mathrm{Sp}} \\
\mathrm{C}_{6} & =2\left[41^{2}\right]_{\mathrm{Sp}} \oplus\left[3^{2}\right]_{\mathrm{Sp}} \oplus[321]_{\mathrm{Sp}} \oplus\left[31^{3}\right]_{\mathrm{Sp}} \oplus\left[2^{2} 1^{2}\right]_{\mathrm{Sp}} \oplus 2[4]_{\mathrm{Sp}} \oplus 3[31]_{\mathrm{Sp}} \oplus 3\left[2^{2}\right]_{\mathrm{Sp}} \\
& \oplus 3\left[21^{2}\right]_{\mathrm{Sp}} \oplus 2\left[1^{4}\right]_{\mathrm{Sp}} \oplus[2]_{\mathrm{Sp}} \oplus 5\left[1^{2}\right]_{\mathrm{Sp}} \oplus 3[0]_{\mathrm{Sp}}
\end{aligned}
$$

Figure 1: The Johnson cokernel in low orders: $C_{3}$ is due jointly to Asada and Nakamura [1] and Hain [8]; $C_{4}$ is due to Morita [14], and the remaining are due to Morita, Sakasai and Suzuki [15].

Theorem There is an epimorphism $C_{s} \rightarrow\left[H^{\langle s\rangle}\right]_{D_{2 s}}$, where the dihedral group acts on $H^{\otimes s}$ in the natural way, twisted by the nontrivial $\mathbb{Z}_{2}$ representation when $s$ is even.

This theorem vastly generalizes the known results for size $s$ representations in $\mathrm{C}_{S}$, which essentially consist of the two series due to Morita and Enomoto and Satoh described above, and of low-order calculations. We show in Theorem 7.3 that both infinite series are contained in $\left[H^{\langle s\rangle}\right]_{D_{2 s}}$. Comparing to computer calculations by Morita, Sakasai and Suzuki [15] shows that $\left[H^{\langle s\rangle}\right]_{D_{2 s}}$ contains all size $s$ representations in $C_{s}$ for $s \leq 6$, which is as far as calculated. A heuristic argument (see Section 7) shows that "most" representations $[\lambda]_{S p}$ appear in $\left[H^{\langle s\rangle}\right]_{D_{2 s}}$. In Theorems 7.5 and 7.6 explicit large infinite families of representations are constructed.

\subsection{Rank 2}

Turning now to $r \geq 2$, let $T(H)$ be the tensor algebra generated by $H$. It is a Hopf algebra with coproduct $\Delta$, antipode $S$ and multiplication $m$. In Section 6 we show that $\Omega_{s-2,2}(H)$ is a quotient of $T(H)^{\otimes 2}$ by certain relations tied to the Hopf algebra structure on $T(H)$ :

Theorem We have

$$
\bigoplus_{s \geq 0} \Omega_{s, 2}(H) \cong\left[T^{+}(H) \otimes T^{+}(H)\right]_{\mathbb{Z}_{2} \times \mathbb{Z}_{2}} / \text { Rel, }
$$

where the $\mathbb{Z}_{2} \times \mathbb{Z}_{2}$ acts via $x \otimes y \mapsto S(x) \otimes S(y)$ and $x \otimes y \mapsto S(y) \otimes S(x)$. The relations $\mathrm{Rel}$ are of the form

(1) $[v, x] \otimes y+x \otimes[v, y]=0$ where $v \in H$,

(2) $(S \otimes m)(\Delta \otimes \mathrm{id})(x \otimes y)+x \otimes y+(m \otimes S)(\mathrm{id} \otimes \Delta)(x \otimes y)=0$. 
Using this presentation to do computer calculations, we find $\Omega_{s-2,2}\langle H\rangle$ for $s \leq 8$ (Theorem 6.2).

\subsection{Comparison to the abelianization of the Lie algebra of symplectic derivations}

Letting $\mathrm{D}_{s}^{\mathrm{ab}}(H)$ be the order $s$ part of the abelianization of $\mathrm{D}^{+}(H)$, Hain's theorem implies that $\mathrm{C}_{s} \rightarrow \mathrm{D}_{s}^{\mathrm{ab}}(H)$ for $s>1$. So the abelianization detects cokernel elements. A theorem of [3] implies that $\Omega_{s+2 r-2, r}\langle H\rangle$ projects onto the rank- $r$ part of the abelianization $\mathrm{D}_{s}^{\mathrm{ab}}(H)$, with the rank defined in the sense of $[4 ; 3]$. The rank-1 part of the abelianization consists of Morita's $[2 m+1]_{\mathrm{Sp}}$ for $m>1$, which does indeed appear in $\Omega_{2 m+1,1}\langle H\rangle$ as noted above. The rank-2 part of the abelianization consists of the following representations [3]: for all $k>\ell \geq 0$,

$$
\begin{gathered}
{[2 k, 2 \ell]_{\mathrm{Sp}} \otimes \mathcal{S}_{2 k-2 \ell+2} \subset \mathrm{D}_{2 k+2 \ell+2}^{\mathrm{ab}}(H),} \\
{[2 k+1,2 \ell+1]_{\mathrm{Sp}} \otimes \mathcal{M}_{2 k-2 \ell+2} \subset \mathrm{D}_{2 k+2 \ell+4}^{\mathrm{ab}}(H),}
\end{gathered}
$$

where $\mathcal{S}_{w}$ and $\mathcal{M}_{w}$ are the vector spaces of weight $w$ cusp forms and modular forms respectively. Hence, these are detected by $\bigoplus_{s} \Omega_{s-2,2}\langle H\rangle$. However $\bigoplus_{s} \Omega_{s-2,2}\langle H\rangle$ contains a lot more, as suggested by the calculations of Theorem 6.2. (See also Conant and Kassabov [2].)

\subsection{Higher rank and future directions}

The spaces $\Omega_{s+2-2 r, r}(H)$ are unwieldy. In [2], we will show there is an epimorphism $\Omega_{s+2-2 r, r}(H) \rightarrow H^{2 r-3}\left(\operatorname{Out}\left(F_{r}\right) ; M_{s+2-2 r, r}\right)$, where $M_{s+2-2 r, r}$ is a certain $\operatorname{Out}\left(F_{r}\right)$-module constructed from the tensor algebra $T(H)$. For rank $r=2$, this implies the following calculations, generalizing the rank- 2 abelianization calculations.

Theorem [2] The space $\oplus_{s} \Omega_{s-2,2}(H)$ surjects onto

$$
\begin{aligned}
& \bigoplus_{k>\ell \geq 0} \mathcal{S}_{2 k-2 \ell+2} \otimes\left(\frac{\mathbb{S}_{(2 k, 2 \ell)}(\mathrm{L})}{\operatorname{ad}(\mathrm{L}) \cdot \mathbb{S}_{(2 k, 2 \ell)}(\mathrm{L})}\right) \\
& \qquad \bigoplus_{k>\ell \geq 0} \mathcal{M}_{2 k-2 \ell+2} \otimes\left(\frac{\mathbb{S}_{(2 k+1,2 \ell+1)}(\mathrm{L})}{\operatorname{ad}(\mathrm{L}) \cdot \mathbb{S}_{(2 k+1,2 \ell+1)}(\mathrm{L})}\right),
\end{aligned}
$$

where $\mathrm{L}=\mathrm{L}(H)$ is the free metabelian Lie algebra on $H$ and $\operatorname{ad}(\mathrm{L})$ is the adjoint action of $\mathrm{L}$ on the Schur functor $\mathbb{S}_{\lambda}(\mathrm{L})$.

The appearance of modular forms and the free metabelian Lie algebra $\mathrm{L}(H)$ in the Johnson cokernel provides yet another connection to number theory which is not yet fully understood. 
Acknowledgements I would like to thank the organizers Nariya Kawazumi and Takuya Sakasai of the 2013 workshop at the University of Tokyo on the Johnson Homomorphism for providing a fertile research environment. I would also like to thank Naoya Enomoto for his talk and subsequent discussion, and Martin Kassabov for insightful comments and discussion. Finally I thank Jetsun Drolma for her inspiration in Yoyogi park.

\section{Basic definitions}

Fix a base field $\mathbb{k}$ of characteristic 0 . Let $\Sigma_{g, 1}$ be a surface of genus $g$ with one boundary component. It has free fundamental group generated by embedded curves $x_{1}, \ldots, x_{g}, y_{1}, \ldots, y_{g}$ with $x_{i}, y_{i}$ intersecting in one point, and all other intersections trivial. Throughout the paper we let $H=H_{1}\left(\Sigma_{g, 1} ; \mathbb{k}\right)$, which is a symplectic vector space. We let $\langle\cdot, \cdot\rangle$ denote the symplectic form, and let $p_{1}, \ldots, p_{g}, q_{1}, \ldots, q_{g}$ be the symplectic basis which is the image of the generating set of the fundamental group. We say $\langle v, w\rangle$ is the contraction of $v$ and $w$. Let $\mathbb{S}_{s}$ be the symmetric group on $s$ letters and for the groups $G \in\left\{\operatorname{Sp}(H), \operatorname{GL}(H), \mathbb{S}_{s}\right\}$, let $[\lambda]_{G}$ be the irreducible representation of $G$ corresponding to $\lambda$.

We begin by defining the relevant Lie algebra which is the target of the Johnson homomorphism.

Definition 2.1 Let $\mathrm{L}_{k}(H)$ be the degree- $k$ part of the free Lie algebra on $H$. Define $\mathrm{D}_{s}(H)$ to be the kernel of the bracketing map $H \otimes \mathrm{L}_{s+1}(H) \rightarrow \mathrm{L}_{s+2}(H)$. Let $\mathrm{D}(H)=\bigoplus_{s=0}^{\infty} \mathrm{D}_{s}(H)$ and $\mathrm{D}^{+}(H)=\bigoplus_{s \geq 1} \mathrm{D}_{s}(H)$. We refer to $s$ as the order of an element of $\mathrm{D}(H)$.

The space $H \otimes \mathrm{L}(H)$ is canonically isomorphic via the symplectic form to $H^{*} \otimes \mathrm{L}(H)$ which is isomorphic to the space of derivations $\operatorname{Der}(\mathrm{L}(H))$. Under this identification, the subspace $\mathrm{D}(H)$ is identified with $\operatorname{Der}_{\omega}(\mathrm{L}(H))=\{X \in \operatorname{Der}(H) \mid X \omega=0\}$, where $\omega=\sum\left[p_{i}, q_{i}\right]$. Thus $\mathrm{D}(H)$ is a Lie algebra with bracket coming from $\operatorname{Der}_{\omega}(H)$.

There is another beautiful interpretation of this Lie algebra in terms of trees:

Definition 2.2 Let $\mathcal{T}(H)$ be the vector space of unitrivalent trees where the univalent vertices are labeled by elements of $H$ and the trivalent vertices each have a specified cyclic order of incident half-edges, modulo the standard AS, IHX and multilinearity relations.(See Figure 2 for the multilinearity relation.) Let $\mathcal{T}_{k}(H)$ be the part with $k$ trivalent vertices. Define a Lie bracket on $\mathcal{T}(H)$ as follows. Given two labeled trees $t_{1}, t_{2}$, the bracket $\left[t_{1}, t_{2}\right]$ is defined by summing over joining a univalent vertex from $t_{1}$ to one from $t_{2}$, multiplying by the contraction of the labels. 
The two spaces $\mathrm{D}_{s}(H)$ and $\mathcal{T}_{s}(H)$ are connected by a map $\eta_{s}: \mathcal{T}_{s}(H) \rightarrow H \otimes \mathrm{L}_{s+1}(H)$ defined by $\eta_{s}(t)=\sum_{x} \ell(x) \otimes t_{x}$ where the sum runs over univalent vertices $x$, $\ell(x) \in H$ is the label of $x$, and $t_{x}$ is the element of $\mathrm{L}_{s+1}(H)$ represented by the labeled rooted tree formed by removing the label from $x$ and regarding $x$ as the root. The image of $\eta_{s}$ is contained in $\mathrm{D}_{s}(H)$ and gives an isomorphism $\mathcal{T}_{s}(H) \rightarrow \mathrm{D}_{s}(H)$ in this characteristic 0 case; see Levine [12].

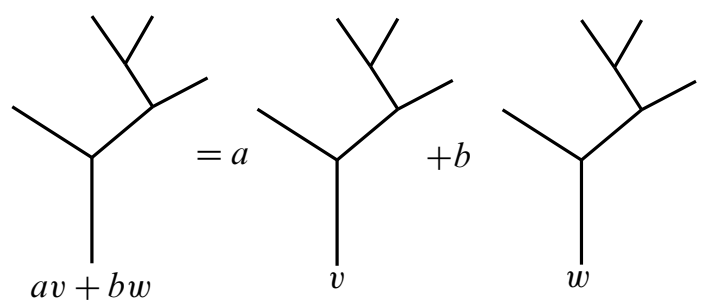

Figure 2: Multilinearity relation in $\mathcal{T}(H)$ : here $a, b \in \mathbb{k}, v, w \in V$

Now that we understand the target of the Johnson homomorphism, we review the construction of the homomorphism itself. Let $F=\pi_{1}\left(\Sigma_{g, 1}\right)$ be a free group on $2 g$ generators and given a group $G$, let $G_{k}$ denote the $k^{\text {th }}$ term of the lower central series $G_{1}=G$ and $G_{k+1}=\left[G, G_{k}\right]$. The Johnson filtration

$$
\operatorname{Mod}(g, 1)=\mathbb{J}_{0} \supset \mathbb{J}_{1} \supset \mathbb{J}_{2} \cdots
$$

of the mapping class group $\operatorname{Mod}(g, 1)$ is defined by letting $\mathbb{J}_{s}$ be the kernel of the homomorphism $\operatorname{Mod}(g, 1) \rightarrow \operatorname{Aut}\left(F / F_{s+1}\right)$. The associated graded $\mathrm{J}_{s}$ is defined by $\mathrm{J}_{s}=\mathbb{J}_{s} / \mathbb{J}_{s+1} \otimes \mathbb{k}$. (The Johnson filtration is a central series, so that the groups $\mathrm{J}_{k}$ are abelian.) Let $J=\bigoplus_{s \geq 1} J_{s}$, where we refer to $s$ as the order of the element.

The group commutator on $\operatorname{Mod}(g, 1)$ induces a Lie algebra structure on $\mathrm{J}$.

It is well known that $\operatorname{Mod}(g, 1) \cong \operatorname{Aut}_{0}(F)$, where

$$
\operatorname{Aut}_{0}(F)=\left\{\varphi \in \operatorname{Aut}(F) \mid \varphi\left(\prod_{i=1}^{g}\left[x_{i}, y_{i}\right]\right)=\prod_{i=1}^{g}\left[x_{i}, y_{i}\right]\right\} \text {. }
$$

Definition 2.3 The (generalized) Johnson homomorphism $\tau: \mathrm{J} \rightarrow \mathrm{D}^{+}(H)$ is defined as follows. Let $\varphi \in \mathbb{J}_{s}$. Then $\varphi$ induces the identity on $\operatorname{Aut}\left(F / F_{S+1}\right)$. Hence for every $z \in F, z^{-1} \varphi(z) \in F_{s+1}$, and we can project to get an element $\left[z^{-1} \varphi(z)\right] \in$ $F_{s+1} / F_{s+2} \otimes \mathbb{k} \cong \mathrm{L}_{s+1}(H)$. Define a map $\tau(\varphi): H \rightarrow \mathrm{L}_{s+1}(H)$ via $z \mapsto\left[z^{-1} \varphi(z)\right]$, where $z$ runs over the standard symplectic basis of $H$. By the various identifications, we can regard $\tau(\varphi)$ as being in $\mathrm{L} \otimes \mathrm{L}_{s+1}(H)$. The fact that $\varphi$ preserves $\prod_{i=1}^{g}\left[x_{i}, y_{i}\right]$ ensures that $\tau(\varphi) \in \mathrm{D}_{s}(H) \subset \mathrm{L} \otimes \mathrm{L}_{s+1}(H)$. 
Proposition 2.4 (Morita) The Johnson homomorphism $\tau: J \rightarrow \mathrm{D}^{+}(H)$ is an injective homomorphism of Lie algebras.

The main object of study of this paper is the Johnson cokernel:

$$
\mathrm{C}_{s}=\mathrm{D}_{s}(H) / \tau\left(\mathrm{J}_{s}\right) .
$$

More precisely, we are interested in the stable part of the cokernel and we always assume that $2 g=\operatorname{dim}(H) \gg s$.

\section{The construction}

We recall from $[3 ; 4]$ the definition of the hairy Lie graph complex and the trace map. The hairy graph complex $C_{k} \mathcal{H}(H)$ is defined as the vector space with basis given by certain types of decorated graphs modulo certain relations.

We begin by describing the generators. Start with a union of $k$ unitrivalent trees with specified cyclic orders at each trivalent vertex. Then join several pairs of univalent vertices by edges, called external edges. The univalent vertices of the trees that were not paired by edges are each labeled by an element of the vector space $H$. These labeled vertices correspond to what are called hairs in [4] and such a graph is called a hairy graph. Note that what we are now calling external edges are called internal edges in [4] to distinguish them from hairs. In the present context, "external" seems more appropriate as these edges are "external" to the trees. In what follows, we will use the graphical convention that external edges are dashed.

Hairy graphs have an orientation, which is defined as a bijection of the trees with the numbers 1 to $k$ and a direction on each external edge.

The relations are

(1) IHX within trees,

(2) AS within trees,

(3) multilinearity on labels of univalent vertices,

(4) switching an edge's direction gives a minus sign,

(5) renumbering the trees gives the sign of the permutation.

These last two types of relations explain how changing the decorations of the graph switches the orientation. Informally $C_{k} \mathcal{H}(H)$ is the space you get by joining $k$ elements 
of $\mathcal{T}(H)$ by several external edges and giving the resulting object an orientation in the above sense.

The boundary operator $\partial: C_{k} \mathcal{H}(H) \rightarrow C_{k-1} \mathcal{H}(H)$ is defined on hairy graphs by summing over joining pairs of distinct trees along external edges. The sign and induced orientation are fixed by the convention that contracting a directed edge from tree 1 to tree 2 induces the orientation where all edge directions are unchanged, the tree formed by joining tree 1 and 2, is numbered 1 and all other tree numbers are reduced by 1 .

In [4], we showed that the abelianization $\mathrm{D}^{\mathrm{ab}}(H)$ embeds in $H_{1}(\mathcal{H}(H))$ via a map which we now define. First, define an operator $T: C_{k} \mathcal{H}(H) \rightarrow C_{k} \mathcal{H}(H)$ by summing over adding an external edge to all pairs of univalent vertices of a hairy graph, fixing the direction arbitrarily and multiplying by the contraction of the two labels. Also define a natural inclusion $\iota: \wedge^{k} \mathcal{T}(H) \rightarrow C_{k} \mathcal{H}(H)$ by regarding $t_{1} \wedge \cdots \wedge t_{k}$ as a union of trees with no external edges. The ordering from the wedge converts to a numbering of the trees as required for the orientation in $C_{k} \mathcal{H}(H)$. Now we can define the trace map from [4].

Definition 3.1 The trace map $\operatorname{Tr}^{\mathrm{CKV}}: \bigwedge^{k} \mathcal{T}(H) \rightarrow C_{k} \mathcal{H}(H)$ is defined as $\operatorname{Tr}^{\mathrm{CKV}}=$ $\exp (T) \circ \iota$.

Unpacking the definition, the trace map $\operatorname{Tr}^{\mathrm{CKV}}$ adds several external edges to a hairy graph in all possible unordered ways. In [4], $\operatorname{Tr}^{\mathrm{CKV}}$ is shown to be a chain map, which is injective on homology, so induces an injection from the abelianization to $H_{1}(\mathcal{H}(H))$.

Now to define $\operatorname{Tr}^{\mathrm{C}}$, consider the subspace $S_{2} \subset C_{2} \mathcal{H}(H)$ consisting of an order-1 tree (tripod) which is connected by two or three of its hairs to the other tree, or has two of its hairs joined by an edge, and the third edge is connected to the other tree. The other tree may have edges connecting it to itself.

Definition 3.2 The target of $\operatorname{Tr}^{\mathrm{C}}$ is defined as $\Omega(H)=C_{1} \mathcal{H}(H) /\left(\partial\left(S_{2}\right)+\iota(\mathcal{T}(H))\right)$.

The $\iota(\mathcal{T}(H))$ term is to eliminate graphs without any edges. Notice that by definition $\Omega(H)$ surjects onto the part of $H_{1}\left(\mathcal{H}_{H}\right)$ with at least one edge. See Figure 3 for a depiction of the three types of relations coming from $\partial\left(S_{2}\right)$. The first kind says that an isolated loop is zero. The second kind says that one can slide a hair along an external edge. The third kind is more complicated, but does not appear until there are at least two external edges attached.

Now we have all the necessary definitions to define the new trace map: 
(1)

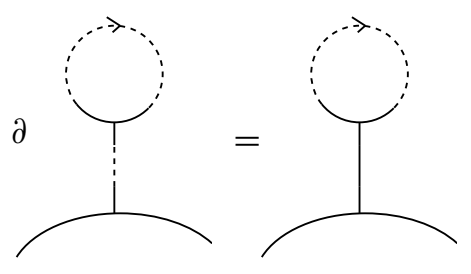

(2)

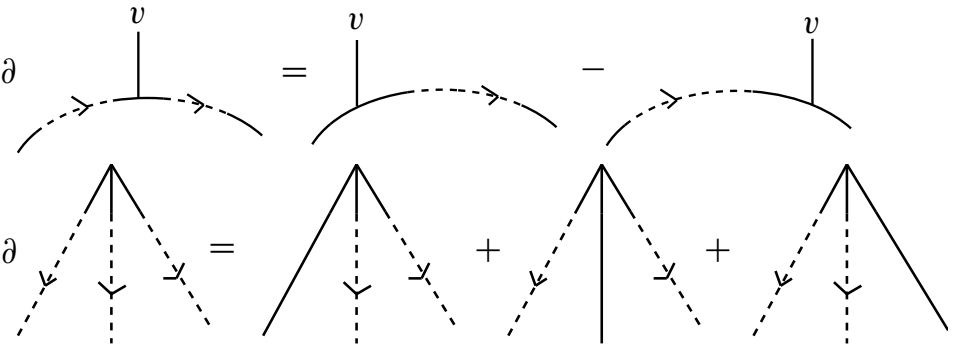

Figure 3: Relations in $\Omega(H)$

Definition 3.3 Define $\operatorname{Tr}^{\mathrm{C}}: \mathcal{T}(H) \rightarrow \Omega(H)$ by the composition:

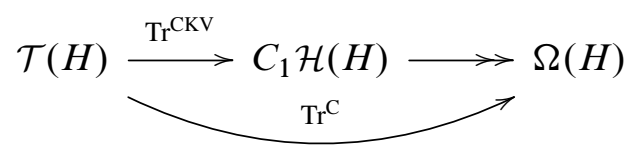

Next we show that $\operatorname{Tr}^{\mathrm{C}}$ is well defined on the cokernel of the Johnson homomorphism.

Theorem 3.4 The map $\operatorname{Tr}^{\mathrm{C}}$ vanishes on the image of the Johnson homomorphism in orders greater than or equal to 2 .

Proof By Hain's theorem, it suffices to show that $\operatorname{Tr}^{\mathrm{C}}([t, X])=0$ if $t$ is of order 1 and $\operatorname{Tr}^{\mathrm{C}}(X)=0$. Indeed, we claim the formula

$$
\operatorname{Tr}^{\mathrm{C}}[t, X]=\left[t, \operatorname{Tr}^{\mathrm{C}}(X)\right]+\left[\operatorname{Tr}^{\mathrm{C}}(t), X\right]
$$

holds. Assume $t$ and $X$ are single trees. The terms of $\operatorname{Tr}^{\mathrm{C}}[t, X]$ come in two types. Those where the added external edges do not join $t$ and $X$ and those where 1 or 2 edges join $t$ and $X$. In the former case, we get the $\left[t, \operatorname{Tr}^{\mathrm{C}}(X)\right]+\left[\operatorname{Tr}^{\mathrm{C}}(t), X\right]$ part we are interested in. If one edge joins $t$ and $X$, we have the situation depicted in Figure 4(1). After applying the trace map, the two indicated terms differ by sliding a hair over an edge, so cancel in $\Omega(H)$. If two hairs join, we have the situation depicted in Figure 4(2), which yields the third $\partial\left(S_{2}\right)$ relation.

So we have shown that $\operatorname{Tr}^{\mathrm{C}}[t, X]=\left[t, \operatorname{Tr}^{\mathrm{C}}(X)\right]+\left[\operatorname{Tr}^{\mathrm{C}}(t), X\right]$. Now $\operatorname{Tr}^{\mathrm{C}}(t)$ is equal to $t$ plus terms where one edge is added. The $t$ is in $\iota(\mathcal{T}(H))$ and therefore is 
(1)
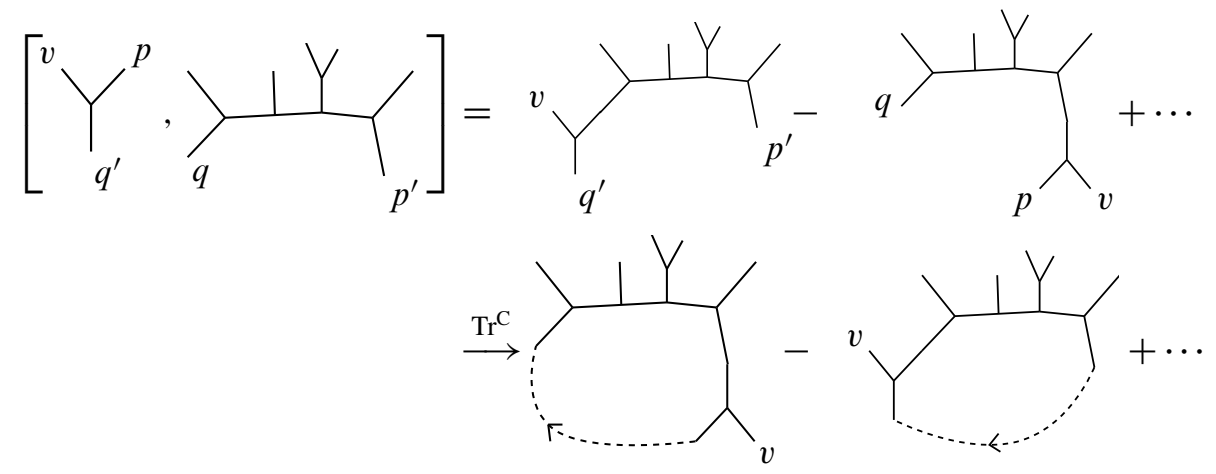

$=\partial\left(S_{2}\right)+\cdots$

(2)

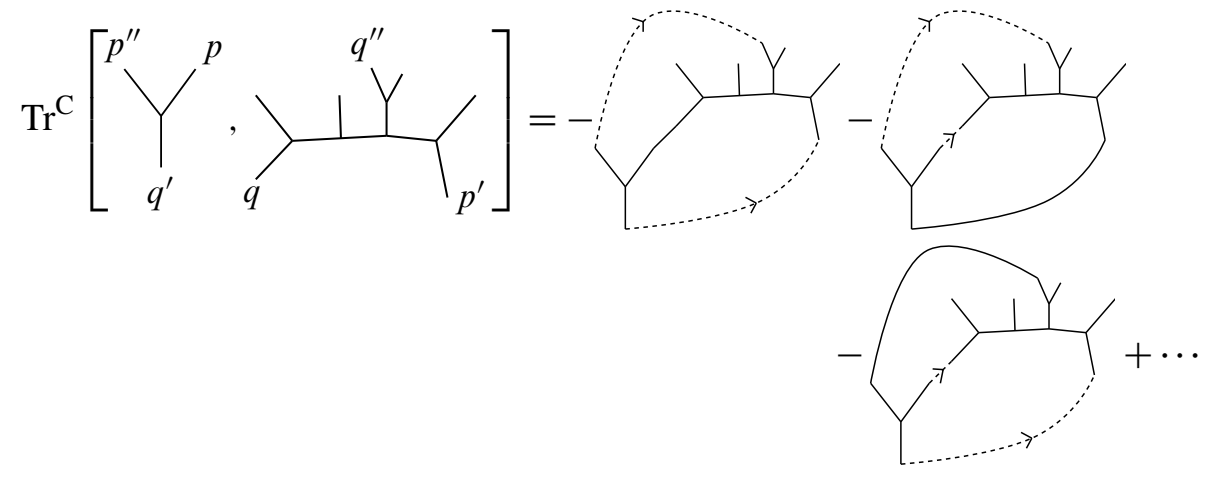

$$
=\partial\left(S_{2}\right)+\cdots
$$

Figure 4: Parts of $\operatorname{Tr}^{\mathrm{C}}[t, X]$ in $\partial\left(S_{2}\right)$

zero. The second type of term is the first kind of $\partial\left(S_{2}\right)$ relation, so is zero. Thus $\operatorname{Tr}^{\mathrm{C}}[t, X]=\left[t, \operatorname{Tr}^{\mathrm{C}} X\right]$, which inductively shows that $\operatorname{Tr}^{\mathrm{C}}$ vanishes on iterated brackets of order-1 elements.

\section{Comparison to the ES-trace}

The space of connected hairy graphs is graded by the first Betti number (rank) and also by number of hairs. Let $C_{1, r, s} \mathcal{H}(H) \subset C_{1} \mathcal{H}(H)$ and $S_{2, r, s} \subset S_{2}$ be the respective subspaces generated by graphs with $b_{1}=r$ and $s$ hairs. Define $\Omega_{s, r}(H)=$ $C_{1, r, s} \mathcal{H}(H) / \partial S_{2, r, s}$. Then

$$
\Omega(H)=\bigoplus_{s \geq 0, r \geq 1} \Omega_{s, r}(H) .
$$

In the next theorem we identify $\Omega_{s, 1}(H)$ with the target of the Enomoto-Satoh trace. 
Theorem 4.1 There is an isomorphism $\Omega_{s, 1}(H) \cong\left[H^{\otimes s}\right]_{D_{2 s}}$ for $s>1$.

Proof Notice that $C_{1,1, s} \mathcal{H}(H)$ is spanned by trees with two univalent vertices joined by an external edge. Using IHX relations, one gets a loop with $s$ labeled hairs attached. Thus $C_{1,1, s} \mathcal{H}(H) \cong\left[H^{\otimes s}\right]_{\mathbb{Z}_{2}}$ where the $\mathbb{Z}_{2}$ acts by reflecting the loop, and has sign $(-1)^{s+1}$. So it gives $v_{1} \otimes \cdots \otimes v_{s} \mapsto(-1)^{s+1} v_{s} \otimes \cdots \otimes v_{1}$. The slide relations have the effect $v_{1} \otimes \cdots \otimes v_{s}=v_{s} \otimes v_{1} \otimes \cdots \otimes v_{s-1}$, giving us $\left[H^{\otimes s}\right]_{D_{2 s}}$. The loop relation is a consequence of IHX and slide relations if $s>1$ :
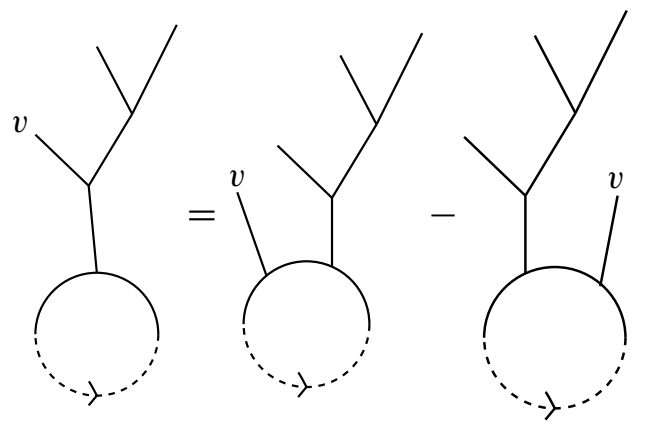

Here any tree can, by IHX, be converted into one of the form $[v, X]$, where $v \in H$, so the picture is sufficiently general. Then the last two terms cancel by a slide relation.

Next we show that $\operatorname{Tr}^{\mathrm{C}}$ projected to $\Omega_{s, 1}(H)$ coincides with the ES-trace. First we show that it possesses an additional $\mathbb{Z}_{2}$-symmetry.

Theorem 4.2 (1) Define $b: H^{\otimes s} \rightarrow H^{\otimes s}$ by $b\left(v_{1} \otimes \cdots \otimes v_{s}\right)=(-1)^{s+1} v_{s} \otimes$ $\cdots \otimes v_{1}$. Then $\operatorname{Tr}^{\mathrm{ES}}: \mathrm{D}_{s}(H) \rightarrow\left[H^{\otimes s}\right]_{\mathbb{Z}_{s}}$ satisfies $b \mathrm{Tr}^{\mathrm{ES}}=\mathrm{Tr}^{\mathrm{ES}}$. Therefore, without loss of information, $\operatorname{Tr}^{\mathrm{ES}}$ takes values in $\left[H^{\otimes s}\right]_{D_{2 s}}$.

(2) The following diagram commutes:

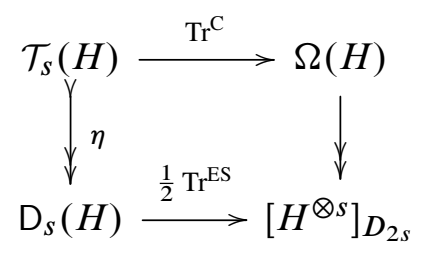

Proof We use the isomorphism $\eta: \mathcal{T}_{S}(H) \rightarrow \mathrm{D}_{s}(H)$. Let $t \in \mathcal{T}_{S}(H)$ be a labeled tree, and consider $\eta(t)=\sum_{x} \ell(x) \otimes t_{x}$. We think of this as a sum of choosing a root for the tree and remembering the label of the root. Satoh's trace map [18] is defined by the embeddings $\mathrm{D}_{s}(H) \hookrightarrow \mathrm{H} \otimes \mathrm{L}_{s+1}(H) \hookrightarrow H \otimes H^{\otimes s+1}$ and then contracting the first two terms to end up in $H^{\otimes s}$. Fix a univalent vertex $x$. Consider what happens if we 
focus on contracting $\ell(x)$ with a label on a fixed univalent vertex of $t_{x}$, say $v$. We can rearrange $t_{x}$ so that $v$ is leftmost, as in the following picture:

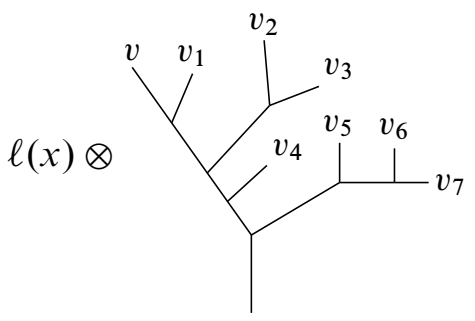

Since we are concentrating on contracting with $v$, we collect all terms in $H^{\otimes(s+1)}$ where $v$ is first. That means that using the relation

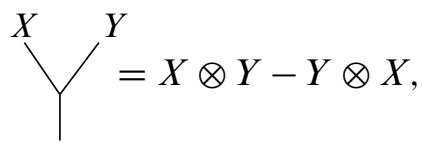

the trees growing off of the arc joining $v$ and the root are expanded in the same order they appear. So for example in the picture above we get $\ell(x) \otimes v v_{1}\left[v_{2}, v_{3}\right] v_{4}\left[v_{5}\left[v_{6}, v_{7}\right]\right]$ which contracts to $\langle\ell(x), w\rangle v_{1}\left[v_{2}, v_{3}\right] v_{4}\left[v_{5}\left[v_{6}, v_{7}\right]\right]$. This is the same element of $H^{\otimes s}$ you would get by adding an edge joining $x$ and the vertex labeled $w$ and read off the word around the cycle running along the direction of the added edge, using the fact that IHX relations near the cycle translate to $[X, Y]=X Y-Y X$. Thus $\operatorname{Tr}^{\mathrm{ES}} \eta(t)$ can be regarded as summing over adding a directed edge between two leaves of the tree, and reading off the resulting word as you run around the cycle. The extra $\mathbb{Z}_{2}$ symmetry comes from the fact that you join two vertices once by an edge running in one direction and once with an edge running in the opposite direction. This reverses the word, and yields a sign of $(-1)^{s+1}$. (One sign for flipping the order of contraction, and $s$ signs for the $s$ trivalent vertices of the tree.) This discussion also shows that $\operatorname{Tr}^{\mathrm{ES}} \eta(t)$ is the same as the 1-edge part of $\operatorname{Tr}^{\mathrm{C}}$. The factor of two arises because we only add one edge for every pair of vertices instead of 2 .

\section{Surjectivity onto a large submodule of $\Omega(H)$}

We begin by defining an analogue of the hairy graph complex and target space $\Omega(H)$ where there is a given bijection from the hairs to $\{1, \ldots, s\}$ as opposed to a labeling of the hairs by vectors.

Definition 5.1 (1) Let $C_{k} \mathcal{H}[s]$ be the space defined analogously to $C_{k} \mathcal{H}(H)$, but instead of labeling the hairs by vectors in $H$, there are $s$ hairs and a fixed 
bijection from these hairs to $1, \ldots, s$. The relations are all the same, except there is no multilinearity. Then $C_{k} \mathcal{H}[s]$ is an $\mathbb{S}_{s}$-module.

(2) Similarly define $S_{2}[s] \subset C_{2} \mathcal{H}[s]$ to be spanned by tripods connected to another tree, by two or three hairs, as well as tripod with a self-loop connected to a tree.

(3) $\Omega[s]$ is defined to be $C_{1} \mathcal{H}[s] /\left(\partial S_{2}[s]+(\right.$ trees with no external edges)).

Notice that we have $C_{k} \mathcal{H}[s] \otimes_{\mathbb{S}_{s}} H^{\otimes s}=\bigoplus_{r} C_{k, r, s} \mathcal{H}(H)$, and $\Omega[s] \otimes_{\mathbb{S}_{s}} H^{\otimes s}=$ $\bigoplus_{r} \Omega_{s, r}(H)$.

Recall that $H^{\langle s\rangle} \subset H^{\otimes s}$ is the intersection of the kernels of all pairwise contractions $H^{\otimes s} \rightarrow H^{\otimes(s-2)}$. Given any partition $\lambda$ of $s$ we recall the following.

\section{Remark 5.2 We have}

(1) $[\lambda]_{\mathbb{S}_{s}} \otimes_{\mathbb{S}_{s}} H^{\otimes s} \cong[\lambda]_{\mathrm{GL}}$,

(2) $[\lambda]_{\mathbb{S}_{s}} \otimes_{\mathbb{S}_{s}} H^{\langle s\rangle} \cong[\lambda]_{\mathrm{Sp}}$,

for $\operatorname{dim}(H)$ large enough compared to $s$. (See the textbook of Fulton and Harris [7] for proofs of these facts.)

Definition 5.3 Define a new complex

$$
C_{k} \mathcal{H}\langle H\rangle=\bigoplus_{s} C_{k} \mathcal{H}[s] \otimes_{\mathbb{S}_{s}} H^{\langle s\rangle},
$$

and a new space

$$
\Omega\langle H\rangle=\bigoplus_{s} \Omega[s] \otimes_{\mathbb{S}_{s}} H^{\langle s\rangle}
$$

By [7], $H^{\otimes s}$ decomposes as a direct sum of Sp-modules, including $H^{\langle s\rangle}$, in a natural way, so there is a projection $H^{\otimes s} \rightarrow H^{\langle s\rangle}$. This gives projections $\pi: C_{k} \mathcal{H}(H) \rightarrow$ $C_{k} \mathcal{H}\langle h\rangle$ and $\pi: \Omega(H) \rightarrow \Omega\langle H\rangle$.

The following theorem is a consequence of a more general theorem of [3].

Theorem 5.4 (Conant, Kassabov and Vogtmann) For dim $H$ large enough compared to $s$,

$$
\pi \circ \operatorname{Tr}^{\mathrm{CKV}}: \mathcal{T}_{s}(H) \rightarrow \bigoplus_{r} C_{1, r, s} \mathcal{H}\langle H\rangle
$$

is an isomorphism.

Corollary 5.5 The composition $\pi \circ \operatorname{Tr}^{\mathrm{C}}: \mathcal{T}_{s}(H) \rightarrow \Omega_{s}\langle H\rangle$ is an epimorphism. 
Proof Consult the commutative diagram

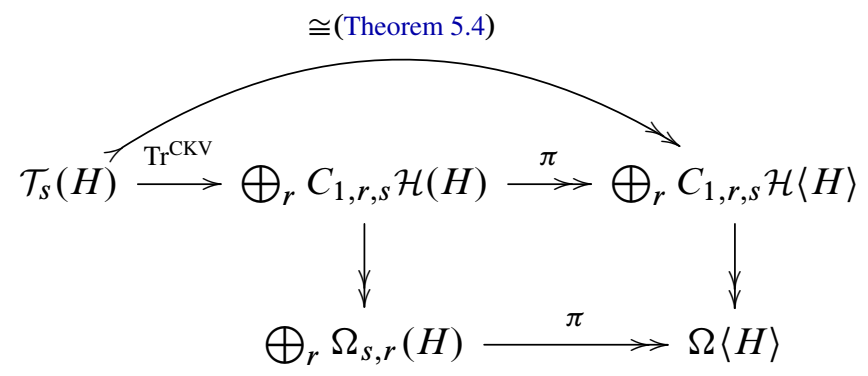

to complete the proof.

Corollary 5.6 In particular $\operatorname{Tr}^{\mathrm{ES}}$ surjects onto $\Omega_{s, 1}\langle V\rangle \cong\left[H^{\langle s\rangle}\right]_{D_{2 s}}$.

Also note that by the above remark if $\Omega_{s, r}(H)=\bigoplus_{\lambda} m_{\lambda}[\lambda]_{\mathrm{GL}}$, then $\Omega_{s, r}\langle H\rangle=$ $\bigoplus_{\lambda} m_{\lambda}[\lambda]_{\mathrm{Sp}}$, so the $\operatorname{GL}(H)$-representation theory for $\Omega(H)$ determines the $\operatorname{Sp}(H)$ representation theory for $\Omega\langle H\rangle$.

\section{Presentation for $\Omega_{s, 2}(H)$}

To set up the main theorem of this section let $T(H)$ be the tensor (free associative) algebra and $T^{+}(H)$ the positive degree part of it. $T(H)$ is a Hopf algebra with antipode $S: T(H) \rightarrow T(H)$ defined by $S\left(v_{1} \cdots v_{k}\right)=(-1)^{k} v_{k} \cdots v_{1}$ for $v_{i} \in H$. For an index set $I=\left\{i_{1}, \ldots, i_{k}\right\}$, let $v_{I}=v_{i_{1}} \cdots v_{i_{k}}$. The coproduct $\Delta: T(H) \rightarrow T(H) \otimes T(H)$ is defined by

$$
\Delta\left(v_{K}\right)=\sum_{K=I \cup J} v_{I} \otimes v_{J}
$$

where the sum is over all partitions of $K$ into two disjoint sets $I$ and $J$. Let $m: T(H) \otimes$ $T(H) \rightarrow T(H)$ be the multiplication operator.

In this section we prove the following theorem:

Theorem 6.1 We have

$$
\bigoplus_{s \geq 0} \Omega_{s, 2}(H) \cong\left[T^{+}(H) \otimes T^{+}(H)\right]_{\mathbb{Z}_{2} \times \mathbb{Z}_{2}} / \text { Rel },
$$

where the $\mathbb{Z}_{2} \times \mathbb{Z}_{2}$ acts via $v_{I} \otimes w_{J} \mapsto S\left(v_{I}\right) \otimes S\left(w_{J}\right)$ and $v_{I} \otimes w_{J} \mapsto S\left(w_{J}\right) \otimes S\left(v_{I}\right)$. The relations Rel are of the form

(1) $\left[v_{0}, v_{I}\right] \otimes w_{J}+v_{I} \otimes\left[v_{0}, w_{J}\right]=0$ where $v_{0} \in H$,

(2) $(S \otimes m)(\Delta \otimes \mathrm{id})\left(v_{I} \otimes w_{J}\right)+v_{I} \otimes w_{J}+(m \otimes S)(\mathrm{id} \otimes \Delta)\left(v_{I} \otimes w_{J}\right)=0$. 
Proof As in the case of $\Omega_{s, 1}$ we can apply IHX relations so that we have a trivalent core graph with hair attached. So we have a unitrivalent tree with all of its univalent vertices joined by external edges in pairs, and to which $s$ hairs are attached. By IHX relations we can move the hair to the edges of the tree that attach to the external edges, and by slide relations we can assume that the hairs are all attached on one side of the external edge. Thus we have two types of generators as depicted in Figure 5. The subscript $e$ stands for "eyeglasses" and the subscript $t$ stands for "theta."

(1) $\left[v_{1} \cdots v_{m} \mid w_{1} \cdots w_{n}\right]_{e}=$

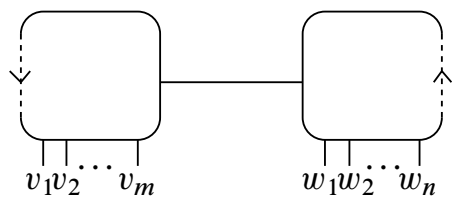

(2)

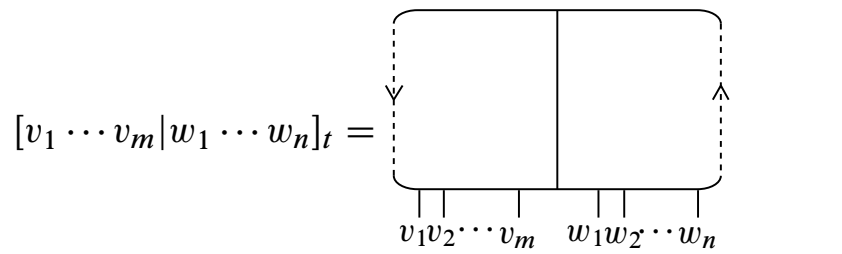

Figure 5: Generators of $\Omega_{s, 2}$ where $m+n=s$

By multilinearity, we may extend the symbols $[x \mid y]_{e, t}$ to any $x, y$ in the tensor algebra $T(H)$. Symmetries of the graphs give rise to the following relations, using the sliding relations to move hairs back to the bottom of the picture (for notational convenience, let $\bar{v}_{I}=S\left(v_{I}\right)$ ):

(S1) $\left[v_{I} \mid w_{J}\right]_{e}=\left[\bar{v}_{I} \mid w_{J}\right]_{e}$.

(S2) $\left[v_{I} \mid w_{J}\right]_{e}=\left[\bar{w}_{J} \mid \bar{v}_{I}\right]_{e}$.

(S3) $\left[v_{I} \mid w_{J}\right]_{t}=\left[\bar{v}_{I} \mid \bar{w}_{J}\right]_{t}$.

(S4) $\left[v_{I} \mid w_{J}\right]_{t}=\left[\bar{w}_{J} \mid \bar{v}_{I}\right]_{t}$.

The loop relation gives us (using IHX)

(L) $\left[\mid w_{J}\right]_{t}=\left[\mid w_{J}\right]_{e}=0$.

The IHX relation has two effects. (IHX1) relates the theta graph and eyeglass graph. However, we also used IHX to push hairs to be near the external edge, and the ambiguity of where to push a hair labeled $v_{0}$ gives (IHX1) below:

(IHX1) $\left[v_{I} v_{0} \mid w_{J}\right]-\left[v_{I} \mid v_{0} w_{J}\right]-\left[v_{0} v_{I} \mid w_{J}\right]+\left[v_{I} \mid w_{J} v_{0}\right]=0$ (e or t) $\operatorname{deg}\left(v_{0}\right)=1$.

$(\mathrm{IHX} 2)\left[v_{I} \mid w_{J}\right]_{e}=\left[v_{I} \mid w_{J}\right]_{t}+\left[\bar{v}_{I} \mid w_{J}\right]_{t}$. 
Finally the boundary of a tripod with three incident edges yields

$$
\text { (TRI) then } \sum_{I \cup J=K}\left[\bar{v}_{I} \mid v_{J} w_{L}\right]_{t}+\left[v_{K} \mid w_{L}\right]_{t}+\sum_{I \cup J=L}\left[v_{K} \bar{w}_{I} \mid w_{J}\right]_{t}=0 \text {. }
$$

To see this consider Figure 6. A boundary is shown in (1). To move the hair off of the left edge of the first summand, we repeatedly use the IHX relation shown in (2), to iteratively build up the terms described in (3).

Using (IHX2) we can express everything in terms of the $t$ generators. (S1) and (S2) are consistent with (S3) and (S4), so we are left with relations (S3), (S4), (L), (IHX1) and (TRI). Interpreting $\left[v_{I} \mid w_{J}\right] \in T(H) \otimes T(H)$ gives the theorem.

Computer calculations using this presentation yield the following results:

Theorem 6.2 For $s \leq 5, \Omega_{s-2,2}(H)=\Omega_{s-2,2}\langle H\rangle=0$, we have:

(1) $\Omega_{4,2}\langle H\rangle \cong\left[1^{4}\right]_{\mathrm{Sp}} \oplus[31]_{\mathrm{Sp}}$, yielding representations in $\mathrm{C}_{6}$.

(2) $\Omega_{5,2}\langle H\rangle \cong 2\left[31^{1}\right]_{\mathrm{Sp}} \oplus\left[2^{2} 1\right]_{\mathrm{Sp}} \oplus\left[21^{3}\right]_{\mathrm{Sp}}$, yielding representations in $\mathrm{C}_{7}$.

(3) $\Omega_{6,2}\langle H\rangle \cong\left[1^{6}\right] \oplus 2[51] \oplus 3[42] \oplus\left[3^{2}\right] \oplus 3[321] \oplus 2\left[2^{3}\right] \oplus 2\left[2^{2} 1^{2}\right] \oplus 2\left[21^{5}\right] \oplus\left[1^{6}\right]$, yielding representations in $\mathrm{C}_{8}$.

\section{Representation theory of $\left[H^{\langle s\rangle}\right]_{D_{2 s}}$}

In this section we analyze the Sp-representation theory of $\left[H^{\langle s\rangle}\right]_{D_{2 s}}$, which is the same as the GL-representation theory of $\left[H^{\otimes s}\right]_{D_{2 s}}$, which can be analyzed via classical Schur-Weyl duality and character theory. Hand calculations with characters yield the following results for low $s$.

Theorem 7.1 (1) We have $\left[H^{\langle 4\rangle}\right]_{D_{8}} \cong\left[21^{2}\right]_{\mathrm{Sp}}$, which picks up the $\left[21^{2}\right]_{\mathrm{Sp}} \in \mathrm{C}_{4}$ found by Morita.

(2) We have $\left[H^{\langle 5\rangle}\right]_{D_{10}} \cong[5]_{\mathrm{Sp}} \oplus[32]_{\mathrm{Sp}} \oplus\left[2^{2} 1\right]_{\mathrm{Sp}} \oplus\left[1^{5}\right]_{\mathrm{Sp}}$. This picks up all of the size $5 \mathrm{Sp}$-representations in $\mathrm{C}_{5}$.

(3) We have $\left[H^{\langle 6\rangle}\right]_{D_{12}} \cong\left[3^{2}\right]_{\mathrm{Sp}} \oplus 2\left[41^{2}\right]_{\mathrm{Sp}} \oplus[321]_{\mathrm{Sp}} \oplus\left[31^{3}\right]_{\mathrm{Sp}} \oplus\left[2^{2} 1^{2}\right]_{\mathrm{Sp}}$. Comparing this to computer calculations of $\mathrm{C}_{6}$ due to Morita, Sakasai and Suzuki [15], this picks up all size 6 representations in $\mathrm{C}_{6}$.

These calculations are suggestive of the following (somewhat optimistic) conjecture:

Conjecture 7.2 All representations of size $s$ in $C_{s}$ are contained in $\left[H^{\langle s\rangle}\right]_{D_{2 s}}$. 
(1)
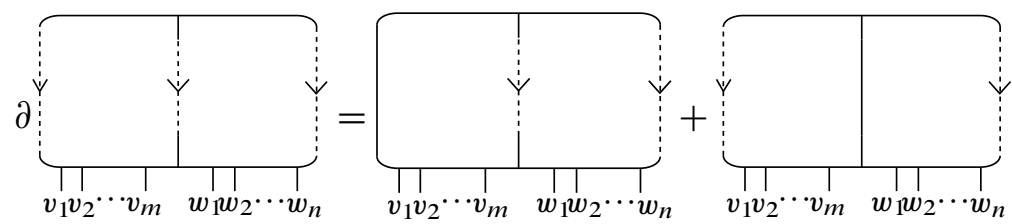

(2)

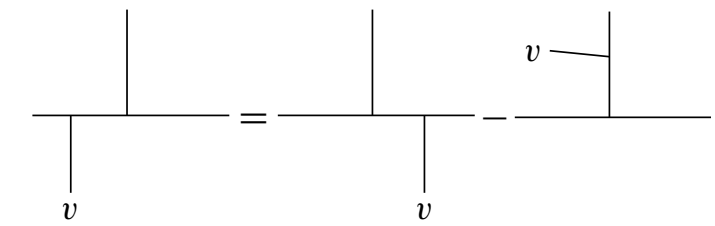

(3)
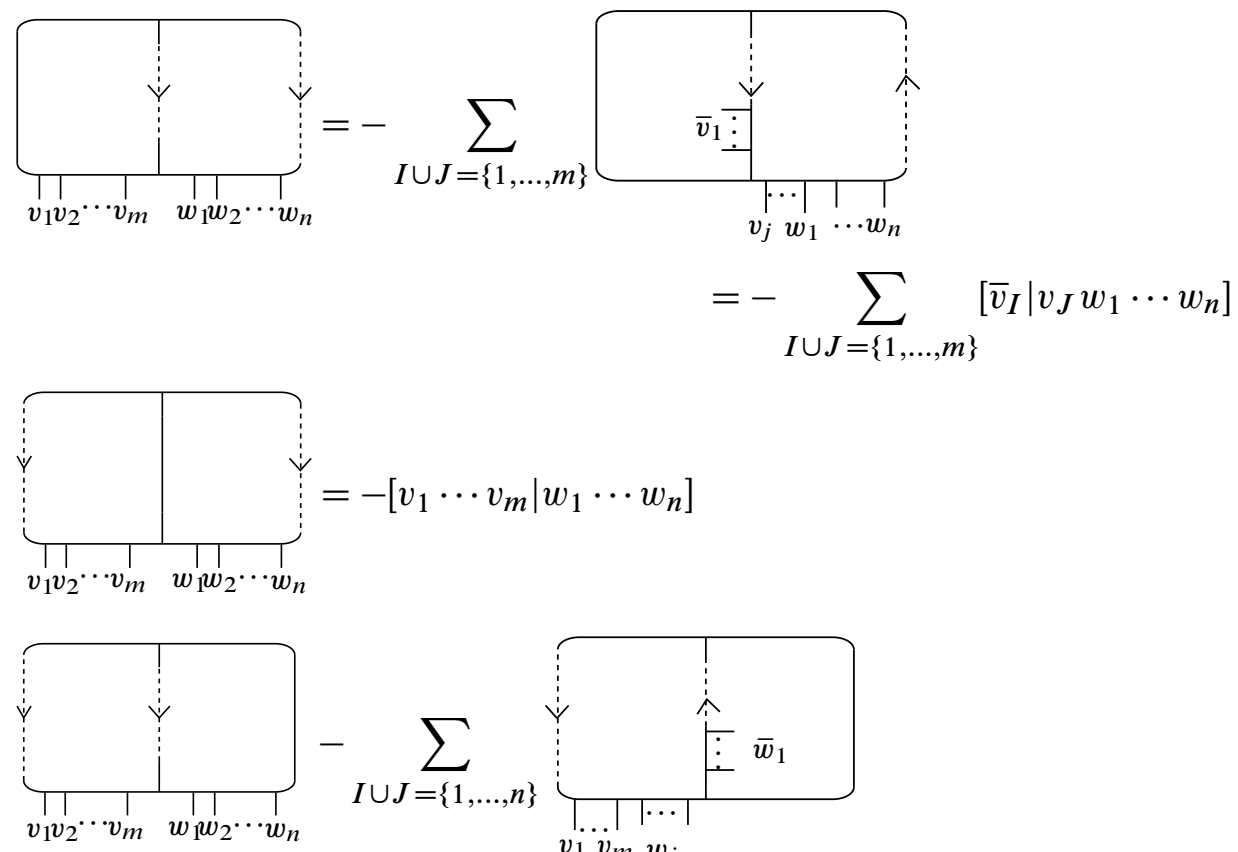

$$
\begin{aligned}
& -\sum_{I \cup J=\{1, \ldots, n\}} \\
& =-\sum_{I \cup J=\{1, \ldots, n\}}\left[v_{1} \cdots v_{m} w_{I} \mid \bar{w}_{J}\right]
\end{aligned}
$$

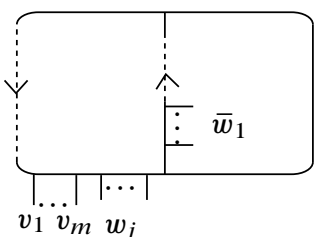

Figure 6: Deriving the TRI relation 
In the next theorem we analyze the 4 representations of lowest complexity, showing that we pick up the Enomoto and Satoh and Morita representations.

Theorem 7.3 (1) The representations $\left[1^{s}\right]_{\mathrm{Sp}}$ only occur when $s=4 m+1$, and in that case with multiplicity one. These are the Enomoto-Satoh terms contained in $\left[H^{\langle 4 m+1\rangle}\right]_{D_{2(4 m+1)}}$.

(2) The representations $[s]_{\mathrm{Sp}}$ only occur when $s=2 m+1$, and in that case with multiplicity one. These are the Morita terms contained in $\left[H^{\langle 2 m+1\rangle}\right]_{D_{2(2 m+1)}}$.

(3) The representations $[s-1,1]_{\mathrm{Sp}}$ and $\left[2,1^{s-2}\right]_{\mathrm{Sp}}$ do not occur in $\left[H^{\langle s\rangle}\right]_{D_{2 s}}$.

Proof For the first statement, it suffices to examine the multiplicity of $\left[1^{s}\right]_{\mathrm{GL}}$ contained in $\left[H^{\otimes(s)}\right]_{D_{2 s}}$. Let $a, b$ be generators of $D_{2 s}$. Then

$$
\begin{gathered}
a \cdot\left(x_{1} \wedge \cdots \wedge x_{s}\right)=x_{2} \wedge \cdots \wedge x_{s} \wedge x_{1}=(-1)^{s-1} x_{1} \wedge \cdots \wedge x_{s}, \\
b \cdot\left(x_{1} \wedge \cdots \wedge x_{s}\right)=(-1)^{s+1} x_{s} \wedge \cdots \wedge x_{1}=(-1)^{s+1+\lfloor s / 2\rfloor} x_{1} \wedge \cdots \wedge x_{s} .
\end{gathered}
$$

So we need $s-1$ and $s+1+\lfloor s / 2\rfloor$ both even, which occurs if and only if $s=4 m+1$. The second statement is proven similarly.

For the third statement, one considers the exact sequences

$$
\begin{aligned}
0 & \rightarrow[s-1,1]_{\mathrm{GL}} \rightarrow S^{s-1}(H) \otimes H \rightarrow S^{s}(H) \rightarrow 0, \\
0 & \rightarrow\left[2,1^{s-2}\right]_{\mathrm{GL}} \rightarrow \bigwedge^{s-1}(H) \otimes H \rightarrow \bigwedge^{s}(H) \rightarrow 0,
\end{aligned}
$$

checking that the $D_{2 s}$ coinvariants of $S^{s-1}(H) \otimes H$ and $\bigwedge^{s-1}(H) \otimes H$ coincide with those of $S^{s}(H)$ and $\bigwedge^{S}(H)$ respectively.

Next we prove a convenient proposition which is instrumental in calculating the $D_{2 s}$ coinvariants of a representation $[\lambda]_{D_{2 s}}$.

Proposition 7.4 In the untwisted case, the coinvariants $\left([\lambda]_{\mathbb{S}_{s}}\right)_{D_{2 s}}$ have dimension

$$
\frac{1}{2 s} \sum_{g \in D_{2 s}} \chi_{\lambda}(g),
$$

where $\chi_{\lambda}$ is the character for $[\lambda]_{\mathbb{S}_{s}}$. In the case where $D_{2 s}$ acts with the $\mathbb{Z}_{2}$ twist, the dimension is

$$
\frac{1}{2 s} \sum_{g \in D_{2 s}} \sigma(g) \chi_{\lambda}(g),
$$

where $\sigma: D_{2 s} \rightarrow\{ \pm 1\}$ maps $a \mapsto 1, b \mapsto-1$. 
Proof Given a character $\chi$ for the dihedral group, define $\int \chi=\frac{1}{2 s} \sum_{g \in D_{2 s}} \chi(g)$. Consulting the character tables for the dihedral group (see James and Liebeck [9, Section 18.3]), for each irreducible character $\chi$, we have

$$
\int \chi= \begin{cases}1 & \text { if } \chi \text { is the character for the trivial representation, } \\ 0 & \text { otherwise. }\end{cases}
$$

So decomposing $[\lambda]_{\mathbb{S}_{s}}$ as a direct sum of irreducible $D_{2 s}$-modules, and writing the character $\chi_{\lambda}$ as a sum of the corresponding dihedral characters, the result follows. The twisted case follows by a similar analysis.

It is a remarkable fact that for symmetric group elements $\sigma$ with large support, $\chi_{\lambda}(\sigma) \ll \chi_{\lambda}(1)$ (see eg Roichman [17] and Larsen and Shalev [11]). Since elements of the dihedral group fix at most two points, this implies that the multiplicities of the $D_{2 n}$ coinvariants appearing in the previous proposition are approximately $\frac{1}{2 n} \chi_{\lambda}(1)=\frac{1}{2 n} \operatorname{dim}\left([\lambda]_{\mathbb{S}_{n}}\right)$. For "most" $\lambda$, we have $\operatorname{dim}[\lambda]_{\mathbb{S}_{n}} \gg 2 n$, and so for such representations $[\lambda]_{\mathbb{S}_{n}}$ appears in $\left[H^{\langle n\rangle}\right]_{D_{2 n}}$ and thus in $C_{n}$. This heuristic argument can be made precise by examining the actual constants involved in the estimates, constructing infinite families of nonzero representations.

As an exercise we work out the exact multiplicities in a couple of different cases. Similar calculations appear in [5].

Theorem 7.5 Let $p \geq 3$ be prime. Let $\alpha_{k}=\left(\begin{array}{c}p \\ k\end{array}\right)-\left(\begin{array}{c}p \\ k-1\end{array}\right)$. If $k>1$ is odd, then $[k, p-k]_{\text {Sp }}$ appears with multiplicity $\alpha_{k} /(2 p)$ in $\mathrm{C}_{p}$. If $k=2 m$, let $\beta_{m}=\left(\begin{array}{c}(p-1) / 2 \\ m\end{array}\right)-$ $\left(\begin{array}{c}p-1) / 2 \\ m-1\end{array}\right)$. Then $[k, p-k]_{\mathrm{Sp}}$ appears with multiplicity $\left(\alpha_{2 m}+\beta_{m}\right) / 2$ in $\mathrm{C}_{p}$.

Proof Given the partition $\lambda=(k, p-k)$, it is easy to calculate $\int \chi$ using the Frobenius character formula. The values of the character on the conjugacy classes $1, a^{r}, b$ are

$$
\begin{aligned}
\chi_{\lambda}(1) & =\left(\begin{array}{l}
p \\
k
\end{array}\right)-\left(\begin{array}{c}
p \\
k-1
\end{array}\right), \\
\chi_{\lambda}\left(a^{r}\right) & = \begin{cases}-1 & k=1, \\
0 & k \geq 2,\end{cases} \\
\chi_{\lambda}(b) & = \begin{cases}0 & k \text { odd }, \\
\left(\begin{array}{c}
(p-1) / 2 \\
m
\end{array}\right)-\left(\begin{array}{c}
(p-1) / 2 \\
m-1
\end{array}\right) & k=2 m .\end{cases}
\end{aligned}
$$

Then $\int \chi_{\lambda}=\frac{1}{2 p}\left(\chi_{\lambda}(1)+(p-1) \chi_{\lambda}\left(a^{r}\right)+p \chi_{\lambda}(b)\right)$, which yields the multiplicities stated in the theorem.

In the next theorem, we consider order $2 p$ where $p$ is prime in order to pick up some even-order representations. Again, for simplicity we restrict to 2 rows. 
Theorem 7.6 Let $p \geq 3$ be prime. For $1<k \leq p$, the representation $[2 p-k, k]_{\mathrm{Sp}}$ appears in $\mathrm{C}_{2 p}$ with multiplicity

$\frac{1}{4 p}\left[\left(\begin{array}{c}2 p \\ k\end{array}\right)-\left(\begin{array}{c}2 p \\ k-1\end{array}\right)+(-1)^{k}(p+1)\left(\begin{array}{c}p \\ m\end{array}\right)-p\left(\begin{array}{c}p-2 \\ m\end{array}\right)+p\left(\begin{array}{c}p-2 \\ m-1\end{array}\right)+2(p-1) \delta_{p, k}\right]$, where $m=\lfloor(k / 2)\rfloor$, and $\delta_{p, k}$ is equal to 0 unless $p=k$, in which case it is 1 .

Proof As in the proof of Theorem 7.5, we calculate $\frac{1}{2(2 p)} \sum_{g \in D_{2(2 p)}} \sigma(g) \chi_{\lambda}(g)$. The conjugacy classes for $D_{2 p}$ and their sizes are written down in Table 1. The dimensions of the $D_{2(2 p)}$ coinvariants are then

$$
\frac{1}{4 p}\left(\chi_{\lambda}(1)+\chi_{\lambda}\left(a^{p}\right)+(p-1) \chi_{\lambda}\left(a^{2 r+1}\right)+(p-1) \chi_{\lambda}\left(a^{2 r}\right)-p \chi_{\lambda}(b)-p \chi_{\lambda}(a b)\right) .
$$

On the symmetric group side, we need to compute $\chi_{\lambda}$ for conjugacy classes of $1, a, a^{2}, a^{p}, b, a b$ where 1 has $2 p$ fixed points, $a$ has $12 p$-cycle, $a^{2}$ has $2 p-$ cycles, $a^{p}$ has $p 2$-cycles, $b$ has $p 2-$ cycles and $a b$ has $p-22-$-cycles and 2 fixed points. Using the Frobenius character formula, we get the values in the chart.

\begin{tabular}{c|cccccc}
$\begin{array}{c}\text { element of } \\
D_{2(2 p)}\end{array}$ & 1 & $a^{p}$ & $\begin{array}{c}a^{r}, \\
r \text { odd }\end{array}$ & $\begin{array}{c}a^{r}, \\
r \text { even }\end{array}$ & $b$ & $a b$ \\
\hline $\begin{array}{c}\text { size of } \\
\text { conjugacy class }\end{array}$ & 1 & 1 & $p-1$ & $p-1$ & $p$ & $p$ \\
\hline$\chi_{[2 p-1,1]}$ & $2 p-1$ & -1 & -1 & -1 & -1 & 1 \\
$\chi_{[2 p-2 m, 2 m]}$ & $\left(\begin{array}{c}2 p \\
2 m\end{array}\right)-\left(\begin{array}{c}2 p \\
2 m-1\end{array}\right)$ & $\left(\begin{array}{c}p \\
m\end{array}\right)$ & 0 & 0 & $\left(\begin{array}{c}p \\
m\end{array}\right)$ & $\left(\begin{array}{c}p-2 \\
m\end{array}\right)-\left(\begin{array}{c}p-2 \\
m-1\end{array}\right)$ \\
$\chi[2 p-2 m-1,2 m+1]$ & $\left(\begin{array}{c}2 p \\
2 m+1\end{array}\right)-\left(\begin{array}{c}2 p \\
2 m\end{array}\right)$ & $-\left(\begin{array}{c}p \\
m\end{array}\right)$ & 0 & 0 & $-\left(\begin{array}{c}p \\
m\end{array}\right)$ & $\left(\begin{array}{c}p-2 \\
m\end{array}\right)-\left(\begin{array}{c}p-2 \\
m-1\end{array}\right)$ \\
$\chi_{[p, p]}$ & $\left(\begin{array}{c}2 p \\
p\end{array}\right)-\left(\begin{array}{c}2 p \\
p-1\end{array}\right)$ & $-\left(\begin{array}{c}p \\
m\end{array}\right)$ & 0 & 2 & $-\left(\begin{array}{c}p \\
m\end{array}\right)$ & $\left(\begin{array}{c}p-2 \\
m\end{array}\right)-\left(\begin{array}{c}p-2 \\
m-1\end{array}\right)$
\end{tabular}

Table 1: Characters for $[2 p-k, k]_{\mathbb{S}_{p}}$ evaluated on conjugacy classes of $D_{2(2 p)}$ : in the last row, suppose $p=2 m+1$.

\section{References}

[1] M Asada, H Nakamura, On graded quotient modules of mapping class groups of surfaces, Israel J. Math. 90 (1995) 93-113 MR1336318

[2] J Conant, M Kassabov, Hopf algebras and invariants of the Johnson cokernel, in preparation

[3] J Conant, M Kassabov, K Vogtmann, Higher hairy graph homology, to appear in Geom. Dedicata

[4] J Conant, M Kassabov, K Vogtmann, Hairy graphs and the unstable homology of $\operatorname{Mod}(g, s), \operatorname{Out}\left(F_{n}\right)$ and $\operatorname{Aut}\left(F_{n}\right)$, J. Topol. 6 (2013) 119-153 MR3029423 
[5] H Enomoto, $\mathbf{N}$ Enomoto, Sp-irreducible components in the Johnson cokernels of the mapping class groups of surfaces, I, Journal of Lie Theory 24 (2014) 687-704

[6] N Enomoto, T Satoh, New series in the Johnson cokernels of the mapping class groups of surfaces, Algebr. Geom. Topol. 14 (2014) 627-669 MR3159965

[7] W Fulton, J Harris, Representation theory, Graduate Texts in Math. 129, Springer, New York (1991) MR1153249

[8] R Hain, Infinitesimal presentations of the Torelli groups, J. Amer. Math. Soc. 10 (1997) 597-651 MR1431828

[9] G James, M Liebeck, Representations and characters of groups, Cambridge Univ. Press (1993) MR1237401

[10] D Johnson, A survey of the Torelli group, from: "Low-dimensional topology", (S J Lomonaco, Jr, editor), Contemp. Math. 20, Amer. Math. Soc. (1983) 165-179 MR718141

[11] M Larsen, A Shalev, Characters of symmetric groups: Sharp bounds and applications, Invent. Math. 174 (2008) 645-687 MR2453603

[12] J Levine, Addendum and correction to: "Homology cylinders: An enlargement of the mapping class group” [Algebr. Geom. Topol. 1 (2001), 243-270], Algebr. Geom. Topol. 2 (2002) 1197-1204 MR1943338

[13] S Morita, Abelian quotients of subgroups of the mapping class group of surfaces, Duke Math. J. 70 (1993) 699-726 MR1224104

[14] S Morita, Structure of the mapping class groups of surfaces: A survey and a prospect, from: "Proceedings of the Kirbyfest", (J Hass, M Scharlemann, editors), Geom. Topol. Monogr. 2 (1999) 349-406 MR1734418

[15] S Morita, T Sakasai, M Suzuki, Slides from presentation at Univ. Tokyo (2013)

[16] H Nakamura, Coupling of universal monodromy representations of Galois-Teichmüller modular groups, Math. Ann. 304 (1996) 99-119 MR1367885

[17] Y Roichman, Upper bound on the characters of the symmetric groups, Invent. Math. 125 (1996) 451-485 MR1400314

[18] T Satoh, On the lower central series of the IA-automorphism group of a free group, J. Pure Appl. Algebra 216 (2012) 709-717 MR2864772

Department of Mathematics, University of Tennessee

227 Ayres Hall, 1403 Circle Drive, Knoxville, TN 37996, USA

jconant@utk.edu

http://www.math.utk.edu/ jconant/

Received: 23 December $2013 \quad$ Revised: 5 July 2014 
